\title{
Telemedicine experiences at a pediatric endocrinology clinic during the COVID-19 pandemic
}

\author{
Yasemin Denkboy Ongen ${ }^{1}$ (C) $\cdot$ Erdal Eren $^{1} \cdot$ Kadriye Cansu Sahin $^{1} \cdot$ Meltem Buhur Pirimoglu $^{1} \cdot$ Halil Saglam $^{1}$. \\ Omer Tarim ${ }^{1}$
}

Received: 3 May 2021 / Accepted: 1 June 2021 / Published online: 7 June 2021

(c) Royal Academy of Medicine in Ireland 2021

\begin{abstract}
Background The coronavirus 19 (COVID-19) pandemic has affected all aspects of life, including the routine follow-up of patients with chronic illnesses. In this study, we aim to share our experience of telemedicine in our pediatric endocrinology clinic during the pandemic.

Methods We were able to continue caring for children with endocrine disorders using various communication methods such as e-mail and e-message.

Results A total of 267 patients received telemedicine care over the course of 608 contacts. The number of hospital visits and physical contact was effectively reduced to help protect against the COVID-19 infection. The patients were supported in terms of receiving their prescriptions and patient education also continued. No complications were observed.

Conclusion The advantages and disadvantages of telemedicine were discussed and consequently, we propose that telemedicine can be utilized to maintain and continue the care of children with endocrine disorders during and even after the pandemic. Further studies are needed to standardize this method for general use.
\end{abstract}

Keywords Children $\cdot$ COVID-19 pandemic $\cdot$ Endocrine disorders $\cdot$ Telehealth $\cdot$ Telemedicine

\section{Introduction}

In early December 2019, the first pneumonia cases of unknown origin were identified in Wuhan City, Hubei Province, China [1]. As a result, a novel beta-coronavirus was named - the 2019 novel coronavirus (2019-nCoV)—which resembled severe acute respiratory syndrome coronavirus (SARS-CoV) [2, 3]. The World Health Organization (WHO) announced that the 2019-nCoV public health emergency was of international concern on January 30 and declared it to be a pandemic on March 11, 2020 [4]. In Turkey, the first positive case was detected on March 11, 2020 [5]. The number

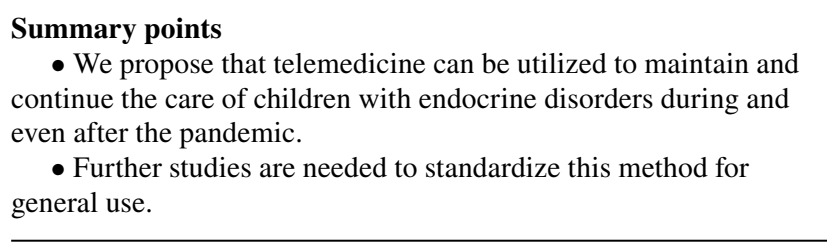

Yasemin Denkboy Ongen

ydenkboyongen@uludag.edu.tr

1 Bursa Uludag University, Department of Pediatric Endocrinology, Bursa, Turkey of cases of COVID-19 in Turkey had been rising daily and hospitals were instructed to receive acute cases only. Over the following days, the government's "stay at home order" for children, and families' fear of the COVID-19, infection led to changes in the delivery of health services. One of these changes was the use of telemedicine. Telemedicine is not novel, long-distance communication for health purposes has a long history. For example, consultation by written correspondence was common from the mid- $1600 \mathrm{~s}$ through to the 1700s [6]. In the following years, telemedicine use developed and became widespread. A search of PubMed from 2010 to 2019 (before the COVID-19 pandemic) using the keywords "telemedicine" and "telehealth" reveals more than 39,636 papers published.

Similar to the rest of the world, telemedicine has evolved in Turkey. It was reported in 2018 that telemedicine was effective in improving the glycemic control of patients with type 1 diabetes mellitus [7]. Telemedicine practice has spread since the COVID-19 pandemic and its advantages and disadvantages have also been reported [8-10]. However, few studies have been published concerning telemedical follow-up for patients with type 1 diabetes mellitus 
[11-14]. Regelmann et al. recently reported the practice of telemedicine and discussed its advantages and disadvantages in a pediatric endocrinology clinic during the COVID-19 pandemic [15].

The aim of this study is to evaluate the impact of telemedicine on pediatric endocrinology patients during the COVID-19 pandemic.

\section{Subjects and methods}

\section{Patients}

A total of 267 patients, in the care of the pediatric endocrinology clinic at Bursa Uludag University Hospital, were included in the study. Data were obtained from electronicmail (google mail, yahoo mail) or electronic message (WhatsApp) records. The study was approved by the local Ethical Committee (approval number: 2020-23/11).

\section{Statistical analysis}

Descriptive analysis was performed using Microsoft Excel for macOS.

\section{Results}

A total of 608 instances of communication were made with 267 patients. The messages were archived in e-mail and e-message boxes. The communications were performed with e-messages with 24 patients and with e-mail for the remainder. Thirty-six patients $(13.4 \%)$ were contacted directly, while 117 patients $(43.8 \%)$ were contacted through the mother, 104 (38.9\%) through the father, and 7 (2.6\%) via a sibling or cousin. In addition, one patient reached us via his family physician and two patients with the assistance of a neighbor (Table 1).

The most common diagnosis was diabetes mellitus (96 patients; $36 \%$ ) (Table 2). The patients' sensor data or glucose measurements on their insulin pump or multiple insulin

Table 1 Communication method of the patients followed up using telemedicine

\begin{tabular}{llll}
\hline Communication method & Yahoo mail & 132 & $49.4 \%$ \\
& Google mail & 111 & $41.6 \%$ \\
& WhatsApp & 24 & $9 \%$ \\
\hline Communicator & Mother & 117 & $43.8 \%$ \\
& Father & 104 & $38.9 \%$ \\
& Self & 36 & $13.4 \%$ \\
& Sibling/cousin & 7 & $2.6 \%$ \\
& Neighbor & 2 & $0.9 \%$ \\
& Family physician & 1 & $0.4 \%$ \\
\hline
\end{tabular}

injections were evaluated, and the insulin dose was adjusted. Diabetes education with particular attention to hypoglycemia and hyperglycemia and sick-day management were updated as required.

The second most common diagnosis was thyroid diseases (56 patients; $20.9 \%$ ). The patients with hypothyroidism (congenital, central, drug-induced, or autoimmune) were followed monthly during the first 6 months of life, bimonthly between 6-12 months, and every 3 months after that with free T4 and TSH measurements obtained by the family physician of the patients. Thyroid function tests followed for patients with thyroid nodules, and the size of the nodules was followed by ultrasound measurement. Patients with hyperthyroidism were also evaluated by analysis the symptoms and free T3, free T4, and TSH measurements obtained from the family physician. During follow-up, the treatment of 3 patients with transient congenital hypothyroidism and two patients with hyperthyroidism were tapered and discontinued. Three newborns referred to us for elevated TSH in the neonatal screening program were examined in our outpatient clinic and their laboratory results were followed. These patients were managed by telemedicine to reduce physical contact. One of the patients was discharged with normal results, while two patients were diagnosed with congenital hypothyroidism, and thyroid hormone replacement was initiated.

Thirty-two (12\%) patients with puberty disorders and 28 patients $(10.5 \%)$ with pituitary diseases were followed in terms of progression of puberty and height and weight percentiles. The patients' complaints and side effects were noted, and the continuity of treatment and support was given in obtaining their prescriptions.

Nineteen patients $(7.1 \%)$ with adrenal diseases were contacted about complaints, laboratory results, compliance to treatment, and drug doses and were also supported with obtaining their prescriptions. Patient education about the management of the adrenal crisis was repeated.

Routine follow-up of the patients with disorders of sexual development (8 patients; $3 \%$ ) and normal variant short stature (10 patients; $3.7 \%$ ) was continued.

Eight patients $(3 \%)$ with obesity were followed in terms of current weight, height, and BMI. Diet and home exercise programs were introduced.

Five patients $(1.9 \%)$ with metabolic bone diseases were contacted about complaints (pain, fracture, deformities). Appointments for bisphosphonate treatment 2 days every 3 months for pamidronate, 1 day every 6 months for zoledronate) were given, and continuity of therapy was maintained with minimum hospital contact.

The diazoxide treatment of a 2-month-old patient with transient hyperinsulinemic hypoglycemia was tapered and discontinued. Another patient with a near-total pancreatectomy due to hyperinsulinemic hypoglycemia on multidrug 


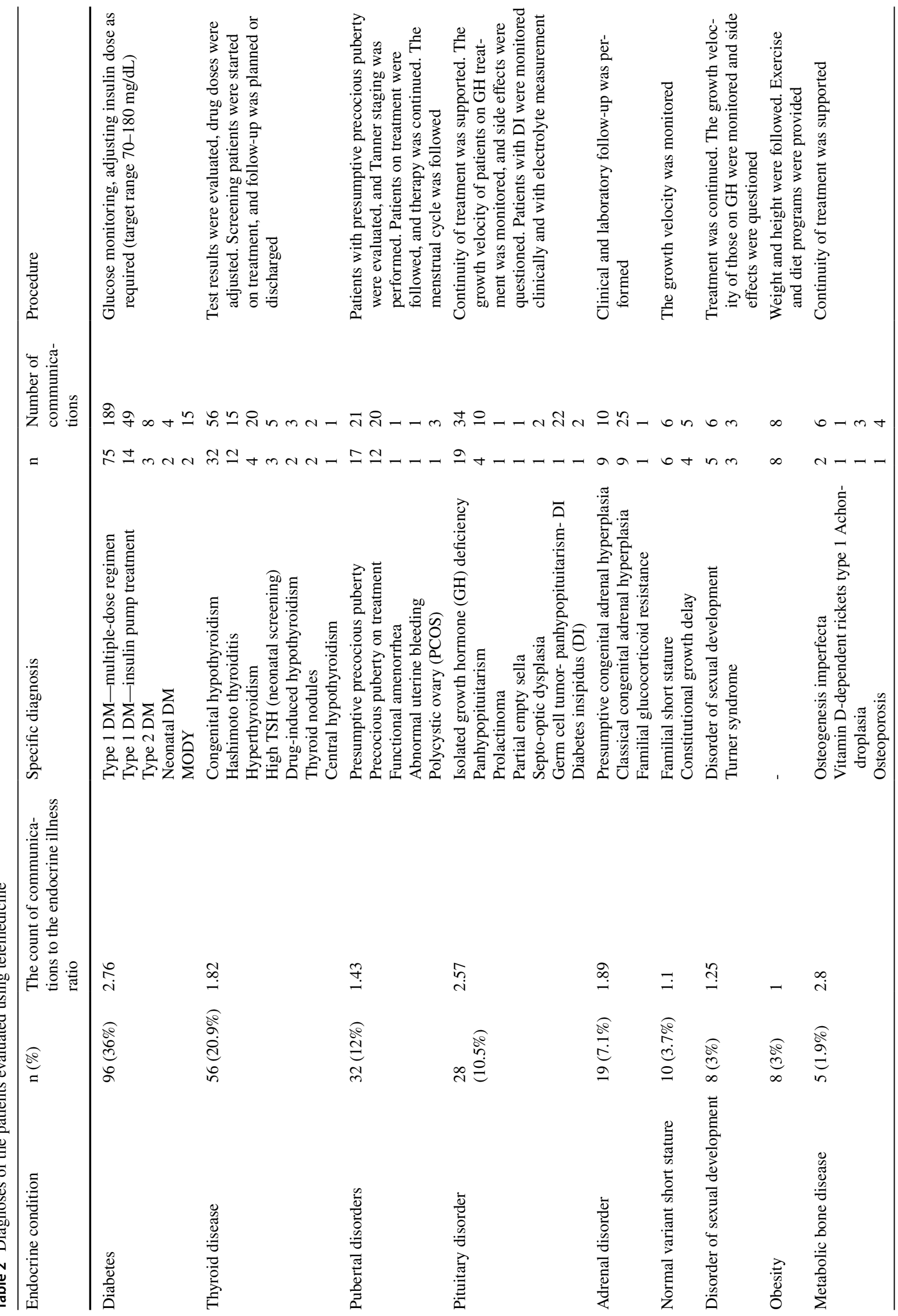


treatment for the maintenance of normoglycemia was followed with the data of continuous glucose monitoring (29 communications). Glucagon and nifedipine were discontinued, and the dose of octreotide was reduced.

The ratio of number of communications to endocrine condition were as follows: hyperinsulinism (9), metabolic bone disease (2.8), diabetes (2.76), pituitary disorders (2.57), thyroid disease (1.82), and pubertal disorders (1.43).

No complication requiring emergency room admission or hospitalization to the clinic or intensive care unit was observed in any of the patient groups. Only one patient acquired COVID-19 PCR positivity due to household contact.

\section{Discussion}

We showed that telemedicine is an effective method for managing pediatric patients with endocrine diseases, especially in certain subgroups (hyperinsulinism, metabolic bone disease, diabetes, pituitary disorders, thyroid disease, and pubertal disorders) without complications. Telemedicine, which has been utilized since 1600 , especially during periods of war and natural disasters, became popular again with the COVID-19 pandemic, which started locally in 2019 and then spread to the entire world $[1,4,6]$. Previous studies have focused on the advantages and disadvantages of telemedicine. Some of the listed disadvantages are a requirement of internet access, insufficient technological ability of the patients, and the inability to perform a physical [8-10]. Regelmann et al. reported that telemedicine provides a practical tool that can be utilized in pediatric endocrinology clinics [15]. We also evaluated the advantages and disadvantages of this method during the COVID-19 pandemic in our clinic (Table 3). Some of our patients who had difficulty using e-mail chose to use e-messages for communication. Those who could not use either method were contacted by telephone or scheduled a face-to-face appointment if necessary. The Italian Pediatric Endocrinology and Diabetes Association recommendations include communication with the health staff by telemedicine, increased use of continuous glucose monitoring (CGM), and continued insulin treatment along with precautions for protection from the COVID infection [16]. Another study on diabetic children and their families reported that the families were satisfied with telemedicine [11]. Our patients were also satisfied with having their regular follow-up by e-mail and e-message without needing to come to the hospital. Other studies similarly reported that face-to-face visits are essential, but technology helps the remote control of diabetes [12]. In a study from Sweden, patients with type 1 diabetes were followed by telemedicine with occasional hospital visits, and they showed an increase in the use of CGM and continuous insulin infusion with a 
Table 3 Advantages and disadvantages of telemedicine in the practice of pediatric endocrinology

\begin{tabular}{|c|c|c|}
\hline & Patient & Health team \\
\hline Advantages & $\begin{array}{l}\text { - Hospital visits were reduced minimizing contact. } \\
\text { - No need to get permission from the work place } \\
\text { - If lab tests are needed, they were performed in the } \\
\text { nearest facility. } \\
\text { - Treatment interruption was prevented. } \\
\text { - Some patients were followed more closely. }\end{array}$ & $\begin{array}{l}\text { - Hospital visits were reduced minimizing contact. } \\
\text { - Follow-up and treatment plans were conveyed by written } \\
\text { messages minimizing misunderstanding. } \\
\text { - Patients who needed to be followed more closely had the } \\
\text { chance of close monitoring. }\end{array}$ \\
\hline Disadvantages & $\begin{array}{l}\text { - Difficult for illiterate patients and relatives } \\
\text { - Internet availability requirement } \\
\text { - Ability to use e-mail and e-message }\end{array}$ & $\begin{array}{l}\text { - Inability to charge the patient due to lack of hospital system. } \\
\text { - Inability to document official record. } \\
\text { - Occasional patient load due to lack of appointment hours. } \\
\text { - Request for communication after working hours. }\end{array}$ \\
\hline
\end{tabular}

decrease in HbA1c [13]. Studies in adult patients with type 1 diabetes have also reported successful diabetes education regarding hypoglycemia, stress, sick-day, ketosis management, and protection from COVID-19 [14].

In our study, telemedicine proved helpful in preventing ketoacidosis and none of our patients were admitted to the emergency room or hospitalized during the pandemic. Furthermore, our patients with multiple pituitary hormone deficiencies or adrenal deficiency complied with their treatment, education for adrenal crisis management was reinforced, and hospital visits were reduced.

\section{Strengths and limitations}

According to national statistics in Turkey, internet access of the population between 16-74 years of age in 2020 was reported at $79 \%$, and the proportion of houses with an internet connection was $90.7 \%$ [17]. This data reveals that internet availability is adequate in our country, and telemedicine can be utilized successfully. Some families were contacted many times. The most important limitations are the lack of an integrated telemedicine system in the hospital, inability to generate an official patient record, and inability to charge the patient. Written communication may prevent misunderstandings, but video conferences may also provide additional useful information.

\section{Conclusions}

In this investigation, we showed that patients living with different chronic endocrine conditions might be followed at home with the aid of local support such as family physicians and pharmacies, their therapy can be managed, and prescriptions can be provided during the pandemic. Although there are many telemedicine studies on patients with type 1 diabetes, there are few studies on patients with other endocrine diseases. Further studies may increase continued use of telemedicine and spread to include routine follow-up even after the pandemic.

Author contribution (I) Conception and design: YDO, EE, OT; (II) administrative support: EE, HS, OT; (III) collection and assembly of data: YDO, KCS, MBP, EE; (IV) data analysis and interpretation: YDO, EE, OT; (V) manuscript writing: all authors; (VI) final approval of manuscript: all authors.

\section{Declarations}

Ethics approval This research study was conducted retrospectively from data obtained for clinical purposes. The study was approved by the local Ethical Committee (approval number: 2020-23/11).

Informed consent All patients provided written informed consent to use their data for scientific purposes.

Conflicts of interest The authors declare no competing interests.

\section{References}

1. Huang C, Wang Y, Li X et al (2020) Clinical features of patients infected with 2019 novel coronavirus in Wuhan. China Lancet 395:497-506. https://doi.org/10.1016/S0140-6736(20)30183-5

2. Lu R, Zhao X, Li J et al (2020) Genomic characterization and epidemiology of 2019 novel coronavirus: implications for virus origins and receptor binding. Lancet 395:565-574. https://doi.org/ 10.1016/S0140-6736(20)30251-8

3. Zhu N, Zhang D, Wang W et al (2020) A novel coronavirus from patients with pneumonia in China, 2019. N Engl J Med 382:727733. https://doi.org/10.1056/NEJMoa2001017

4. https://covid19.who.int Accessed: 03.13.2021.

5. https://covid19.saglik.gov.tr/Eklenti/39551/0/covid-19rehberig enelbilgilerepidemiyolojivetanipdf.pdf Access date: 03.13.2021.

6. Ryu S (2010) History of telemedicine: evolution, context, and transformation. Healthc Inform Res 16(1):65-66. https://doi.org/ 10.4258/hir.2010.16.1.65

7. Döğer E, Bozbulut R, Soysal Acar AŞ et al (2019) Effect of telehealth system on glycemic control in children and adolescents with type 1 diabetes. J Clin Res Pediatr Endocrinol 11(1):70-75. https://doi.org/10.4274/jcrpe.galenos.2018.2018.0017

8. Wijesooriya NR, Mishra V, Brand PLP et al (2020) COVID-19 and telehealth, education, and research adaptations. Paediatr Respir Rev 35:38-42. https://doi.org/10.1016/j.prrv.2020.06.009 
9. Barney A, Buckelew S, Mesheriakova V et al (2020) The COVID19 Pandemic and rapid implementation of adolescent and young adult telemedicine: challenges and opportunities for innovation. J Adolesc Health 67(2):164-171. https://doi.org/10.1016/j. jadohealth.2020.05.006

10. Mahajan V, Singh T, Azad C (2020) Using telemedicine during the COVID-19 pandemic. Indian Pediatr 57(7):652-657. https:// doi.org/10.1007/s13312-020-1895-6

11. Odeh R, Gharaibeh L, Daher A et al (2020) Caring for a child with type 1 diabetes during COVID-19 lockdown in a developing country: challenges and parents' perspectives on the use of telemedicine. Diabetes Res Clin Pract 168:108393. https://doi. org/10.1016/j.diabres.2020.108393

12. Fuchs J, Hovorka R (2020) COVID-19 and diabetes: could diabetes technology research help pave the way for remote healthcare? J Diabetes Sci Technol 14(4):735-736. https://doi.org/10.1177/ 1932296820929714

13. Ludvigsson J (2020) Effect of COVID-19 pandemic on treatment of type 1 diabetes in children. Acta Paediatr 00:1-2. https://doi. org/10.1111/apa.15627
14. Ghosh A, Gupta R, Misra A (2020) Telemedicine for diabetes care in India during COVID19 pandemic and national lockdown period: guidelines for physicians. Diabetes Metab Syndr 14(4):273-276. https://doi.org/10.1016/j.dsx.2020.04.001

15. Regelmann MO, Conroy R, Gourgari E et al (2020) pediatric endocrinology in the time of COVID-19: considerations for the rapid implementation of telemedicine and management of pediatric endocrine conditions. Horm Res Paediatr 93(6):343-350. https://doi.org/10.1159/000513060

16. d'Annunzio G, Maffeis C, Cherubini V et al (2020) Caring for children and adolescents with type 1 diabetes mellitus: Italian Society for Pediatric Endocrinology and Diabetology (ISPED) statements during COVID-19 pandemia. Diabetes Res Clin Pract 168:108372. https://doi.org/10.1016/j.diabres.2020.108372

17. https://data.tuik. gov.tr/Bulten/Index $? \mathrm{p}=$ Hanehalki-BilisimTeknolojileri-(BT)-Kullanim-Arastirmasi-2020-33679 Accessed: 03.13.2021 